\title{
Stimulus control following training on a conditional discrimination
}

\author{
RALPH W. RICHARDS \\ Colorado State University, Fort Collins, Colorado 80523
}

\begin{abstract}
Pigeons were trained on a conditional discrimination in which responding was reinforced either in the presence or in the absence of a vertical line, depending on the color of the key and the chamber's illumination. When subsequently tested for generalization along the line-orientation dimension, all subjects showed a decremental gradient when the lines were combined with the color that had signaled reinforcement in the presence of the vertical line. In addition, most subjects showed an incremental gradient when the lines were combined with the color that had signaled no reinforcement in the presence of the vertical line.
\end{abstract}

The orientation of a line typically gains control over pigeons' responding following training on a multiple variable-interval extinction (mult VI EXT) schedule in which the key is successively illuminated by a homogeneous light or by a line on an otherwise identical background. If the presence of the line is associated with the VI component, a generalization test typically produces a decremental gradient (e.g., Hearst, 1968; Honig, Boneau, Burstein, \& Pennypacker, 1963). On the other hand, if the presence of the line is associated with the EXT component, an incremental generalization gradient typically is obtained (e.g., Farthing \& Hearst, 1968; Hearst, 1968; Honig, Boneau, Burstein, \& Pennypacker, 1963).

Boneau and Honig (1964), however, failed to obtain such gradients after training pigeons on a fourcomponent multiple schedule in which the presence (or absence) of a line was associated with either a VI or EXT schedule, depending on the color of the key's background. More specifically, pigeons were trained on a conditional discrimination in which responding to a vertical line on a 550-nm background or to a plain $570-\mathrm{nm}$ background was reinforced and responding to either a plain 550-nm background or a vertical line on a 570-nm background was not reinforced. Although subjects learned the discrimination, a subsequent generalization test revealed little evidence of control by the line's orientation regardless of background color on the key. That is, subjects responded at essentially the same high rate when the background was a $550-\mathrm{nm}$ light and at essentially the same relatively low rate when the background was a $570-\mathrm{nm}$ light, regardless of the orientation of the line. Boneau and Honig hypothesized that the difference between their results and those typically obtained fol-

Reprints may be obtained from the author, Department of Psychology, Colorado State University, Fort Collins, Colorado 80523. lowing training on a two-component multiple schedule (a so-called simple discrimination) could have been due to the greater demands that their fourcomponent multiple schedule (conditional discrimination) placed on the subjects' information processing capabilities. They suggested that "the attention that the animal will pay to nonessential or irrelevant features [the line's orientation] of the stimulus situation is a function of the demands made on it to process other information. When these demands are not great, as in the simple discrimination, nonessential features have a chance to control behavior; but they will not do so when the animal's capacity to process information is taxed severely by relevant features [presence or absence of the line plus color of the background] of the stimulus situation" (p. 93). Sutherland and Mackintosh (1971) take a similar position in suggesting that the "results [of Boneau \& Honig] are consistent with the idea that there is a limit to the number of cues to which animals are capable of attending. When animals are only required to switch-in an analyzer detecting the presence or absence of a vertical line, they are likely also to switch-in an analyzer detecting its orientation (indeed, they are more likely to do so under these circumstances, than if given nondifferential training). However, when they are required both to switch-in an analyzer detecting the presence of a line and another analyzer to detect the color of the background, they are unlikely to switch-in an orientation analyzer strongly" (pp. 233-234).

In light of the theoretical importance attributed to the Boneau and Honig study, it is surprising that the reliability of their results apparently has not been examined. Thus, the major purpose of the present study was to determine whether the absence of stimulus control along the irrelevant dimension of line orientation is a general outcome following training on a conditional discrimination. To this end, pigeons were exposed to a training and testing regimen some- 
what different from that employed by Boneau and Honig.

\section{METHOD}

\section{Subjects}

Six adult, female, White Carneaux pigeons were maintained at approximately $80 \%$ of their free-feeding weights. All subjects were previously trained on a multiple schedule involving immediate and delayed reinforcement, but no subject had been exposed to a line or to any type of geometric figure projected on the key. Two other subjects were discarded because they showed little evidence of discrimination learning after 30 days of training.

\section{Apparatus}

A Kodak slide projector was mounted behind the center key of a three-key operant conditioning chamber (internal dimensions: $30.5 \mathrm{~cm}$ long $\times 35 \mathrm{~cm}$ wide $\times 35 \mathrm{~cm}$ high). During training, the center key was illuminated by a homogeneous white light (W), a homogeneous red light $(R)$, or a black vertical line (approximately $2 \mathrm{~mm}$ thick) on either the white (LW) or red (LR) background. During the generalization test, five other orientations of the line (departures of $+30^{\circ},+60^{\circ},-30^{\circ},-60^{\circ}$, and $+90^{\circ}$ from vertical) were presented on the white or red surround. In both training and testing, a white houselight on the front wall provided general illumination when the key contained a white surround, and a red pilot light on the back wall provided illumination when the key contained a red surround.

Standard electromechanical equipment was located in an adjacent room.

\section{Procedure}

Because of their prior history, the birds were exposed immediately to the conditional discrimination. Throughout this training, a multiple VI 30-sec VI 30-sec EXT EXT schedule was in operation. The keypecking of Birds 1, 2, and 3 was reinforced during $R$ and $L W$ and not reinforced during $L R$ and $W$. The keypecking of Birds 4, 5, and 6 was reinforced during $L R$ and $W$ and not reinforced during $R$ and $L W$. The reinforcer was $3 \mathrm{sec}$ access to mixed grain; the timing of this interval began when the subject placed its head into the food aperture and interrupted a light beam focused on a photocell.

Sessions 1-55 contained 71 -min presentations of each training stimulus, and Sessions 56-75 and 77-81 contained 15 30-sec presentations of each training stimulus. The order of presentation was mixed, and successive stimuli were separated by a 1-sec darkening of the chamber. Available reinforcements not collected during one stimulus presentation carried over to the next presentation of that stimulus.
Sessions 76 and 82 began with a period of additional training (30 stimulus presentations) and ended with a generalization test that was conducted in extinction. The first generalization test employed the seven stimuli involving the red surround and red pilot light, and the second generalization test employed the seven stimuli involving the white surround and white houselight. In both tests, each test stimulus was presented once in each of seven blocks. Throughout, stimuli were presented for $30 \mathrm{sec}$ and separated by a 1-sec darkening of the chamber.

\section{RESULTS}

Table 1 presents each bird's mean response rate to the training stimuli during the five sessions immediately prior to the two generalization tests. The table also shows the proportion of responses emitted in the presence of the red (and white) lights during the stimulus associated with reinforcement. By the end of training, all birds were responding more rapidly during the stimuli associated with reinforcement than with extinction. There were, however, large individual differences in the accuracy and consistency of the birds' performance. For example, B-2, -3 , and -4 showed essentially the same high level of accuracy regardless of the color of the key or chamber's illumination and regardless of whether the line was associated with the VI or EXT schedule. But B-1, -5, and -6 performed better when the key and chamber were illuminated red; the absence of the line on red was associated with the VI schedule for B-1 and with EXT for B-5 and B-6.

The results of the generalization tests involving the red background are shown in Figure 1. The numbers in parentheses near each gradient are, in order, the number of responses emitted to the key when it contained the vertical line $\left(0^{\circ}\right)$ and when it contained no line during the generalization test. The generalization gradients from B-1, -2 , and -3 are U-shaped and have their nadirs located at or near the $0^{\circ}$ line (associated with EXT during training). The gradients from B-4, -5 , and -6 are shaped like an inverted $U$ and peak at or near the $0^{\circ}$ line (associated with the VI schedule during training).

Table 1

Absolute Response Rates (Responses/Minute) to Each Stimulus and Relative Response Rates to the Stimuli Associated With Reinforcement During the Five Sessions Immediately Prior to the Generalization Tests

\begin{tabular}{|c|c|c|c|c|c|c|c|c|c|c|c|c|}
\hline \multirow[t]{2}{*}{ Bird } & \multicolumn{5}{|c|}{ Sessions $71-75$} & \multicolumn{7}{|c|}{ Sessions $76-81$} \\
\hline & $\mathbf{R}$ & LR & $\frac{R}{R+L R}$ & LW & $\mathbf{W}$ & $\frac{L W}{L W+W}$ & $\mathbf{R}$ & LR & $\frac{R}{R+L R}$ & LW & W & $\begin{array}{c}L W \\
L W+W\end{array}$ \\
\hline $\begin{array}{l}\text { B-1: } \\
\text { B-2: } \\
\text { B-3: }\end{array}$ & $\begin{array}{r}124 \\
102 \\
37\end{array}$ & $\begin{array}{r}15 \\
6 \\
5\end{array}$ & $\begin{array}{l}.89 \\
.95 \\
.87\end{array}$ & $\begin{array}{r}109 \\
92 \\
57\end{array}$ & $\begin{array}{r}36 \\
3 \\
11\end{array}$ & $\begin{array}{l}.75 \\
.97 \\
.84\end{array}$ & $\begin{array}{r}115 \\
99 \\
42\end{array}$ & $\begin{array}{l}9 \\
6 \\
3\end{array}$ & $\begin{array}{l}.93 \\
.95 \\
.93\end{array}$ & $\begin{array}{r}101 \\
87 \\
60\end{array}$ & $\begin{array}{r}50 \\
1 \\
5\end{array}$ & $\begin{array}{l}.67 \\
.99 \\
.92\end{array}$ \\
\hline & LR & $\mathbf{R}$ & $\frac{\mathrm{LR}}{\mathrm{LR}+\mathrm{R}}$ & W & LW & $\frac{W}{W+L W}$ & LR & $\mathbf{R}$ & $\frac{L R}{L R+R}$ & W & LW & $\begin{array}{c}W \\
W+L W\end{array}$ \\
\hline $\begin{array}{l}\text { B-4: } \\
\text { B-5: } \\
\text { B-6: }\end{array}$ & $\begin{array}{l}57 \\
88 \\
54\end{array}$ & $\begin{array}{r}3 \\
10 \\
6\end{array}$ & $\begin{array}{l}.96 \\
.89 \\
.90\end{array}$ & $\begin{array}{r}70 \\
82 \\
+\quad 55\end{array}$ & $\begin{array}{r}2 \\
27 \\
13\end{array}$ & $\begin{array}{l}.98 \\
.75 \\
.81\end{array}$ & $\begin{array}{l}57 \\
81 \\
54\end{array}$ & $\begin{array}{r}4 \\
10 \\
5\end{array}$ & $\begin{array}{l}.94 \\
.89 \\
.92\end{array}$ & $\begin{array}{l}71 \\
74 \\
54\end{array}$ & $\begin{array}{r}2 \\
31 \\
9\end{array}$ & $\begin{array}{l}.97 \\
.70 \\
.86\end{array}$ \\
\hline
\end{tabular}




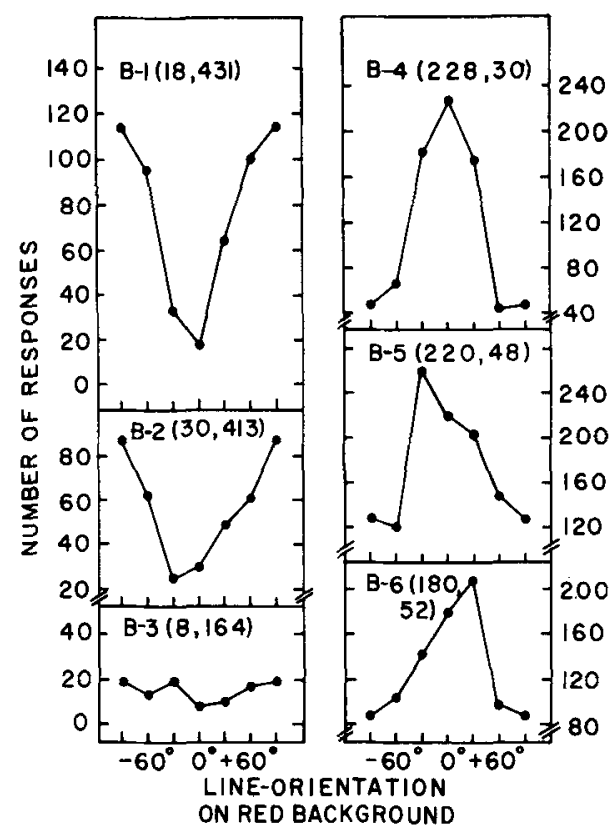

Figure 1. Total number of responses emitted to each of the generalization test stimuli when the key and chamber were illuminated by a red light. For the sake of symmetry, the number of responses to the horizontal line has been plotted at $+90^{\circ}$ and $-90^{\circ}$ for each subject. The numbers in parentheses near each gradient are, in order, the number of responses to the key when it contained the vertical line and no line during the generalization test.

Figure 2 shows the generalization gradients obtained when the test stimuli were combined with the white background. The gradients from B-1, -2 , and -3 are shaped like an inverted $U$ and peak at the $0^{\circ}$ line (associated with the VI schedule during training). However, there is tremendous variability in the shape of the gradients of the remaining birds, which had this $0^{\circ}$ line associated with EXT during training. B-4 showed a gradient shaped like a U, but B-5 showed a gradient shaped like an inverted $U$. The gradient for B-6 was irregular.

\section{DISCUSSION}

The present results showed that the line's orientation did control subjects' responding after they were trained on a conditional discrimination in which the presence or absence of the line was associated with reinforcement or extinction, depending on the color of the key and chamber's illumination. The six birds showed decremental generalization gradients when the line orientations were combined with the color that had signaled reinforcement in the presence of the vertical line. Four of the birds also showed incremental gradients when the line orientations were combined with the color that had signaled extinction in the presence of the vertical line. The two subjects that did not show incremental gradients also showed relatively poor discrimination between the training stimuli prior to and during the generalization test. Whether this or some other factor (such as the prior generalization test) was responsible for their not showing incremental gradients is not clear. In any event, to the extent that a generalization gradient's shape is a valid index of excitatory and inhibitory control [as suggested by Jenkins (1965), Rilling (1977), and Terrace (1972); for a recent discussion of this matter, also see Richards \& Marcattilio (1978)], it may be concluded that the vertical line functioned as an excitatory stimulus when compounded with one color (for all six birds), and as an inhibitory stimulus when compounded with the other color (for four birds).

The present study thus shows that the Boneau and Honig (1964) finding has limited generality. Apparently, something in their training or testing procedure prevented them from obtaining control by the line's orientation. While an empirical search for the factor or factors responsible for Boneau and Honig's results was not undertaken, several differences between the procedures of the twe studies are readily apparent. For example, the cues that signaled whether the line was associated with reinforcement or extinction were probably more similar in Boneau and Honig's study than in the present study. In the Boneau and Honig study, whether the vertical line was associated with

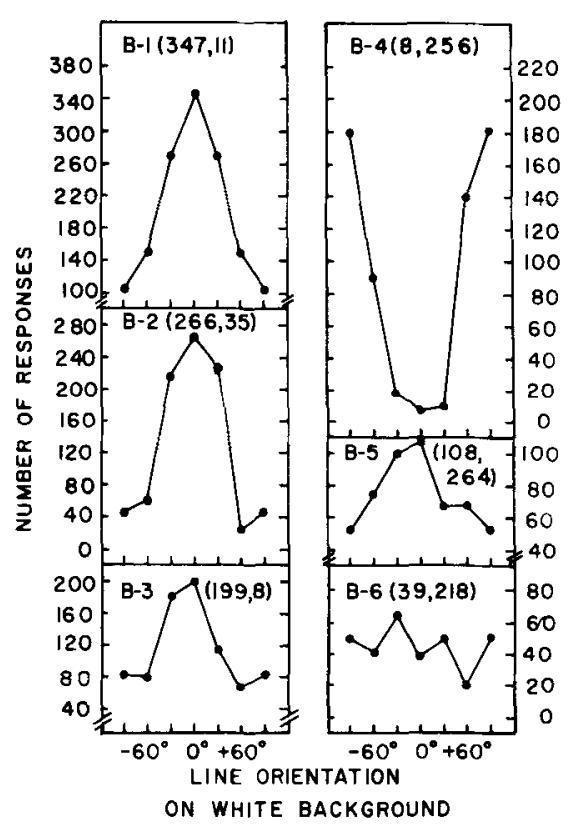

Figure 2. Total number of responses emitted to each of the generalization test stimuli when the key and chamber were illuminated by a white light. For the sake of symmetry, the number of responses to the horizontal line has been plotted at $+90^{\circ}$ and $-90^{\circ}$ for each subject. The numbers in parentheses near each gradient are, in order, the number of responses to the key when it contained the vertical line and no line during the generalization test. 
reinforcement or extinction depended on whether the key was illuminated by wavelengths that differed by only $20 \mathrm{~nm}$; in the present study, it depended on whether the key and chamber was illuminated by a red or a white light. It may also be important to note that in the Boneau and Honig study, the line orientations were presented on both colors in the same generalization session, but that in the present study the line orientations were compounded with only one color in a generalization test session. Since previous research (e.g., Thomas, Svinicki, \& Svinicki, 1970) has shown that some generalization test procedures may mask the existence of stimulus control along the line-orientation dimension, it may be that the Boneau and Honig results were due to their using an insensitive testing procedure.

Regardless of the factor or factors responsible for the different results obtained in the two studies, it seems clear that a conditional discrimination does not necessarily overload the information-processing capabilities of the pigeon and prevent the irrelevant aspect of a line's orientation from gaining control over behavior. In the more theoretical and speculative terminology of Sutherland and Mackintosh (1971), it would be suggested that pigeons may switch in an orientation analyzer even after presumably switching in one analyzer to detect the presence of the line and another analyzer to detect the color by which the key and/or chamber are illuminated.

\section{REFERENCES}

Boneau, C. A., \& Honig, W. K. Opposed generalization gradients based upon conditional discrimination training. Journal of Experimental Psychology, 1964, 66, 89-93.

Farthing, G. W., \& Hearst, E. Generalization gradients of inhibition after different amounts of training. Journal of the Experimental Analysis of Behavior, 1968, 11, 743-752.

Jenkins, H. M. Generalization gradients and the concept of inhibition. In D. Mostofsky (Ed.), Stimulus generalization. Stanford: Stanford University Press, 1965.

Hearst, E. Discrimination learning as the summation of excitation and inhibition. Science, 1968, 162, 1303-1306.

Honig, W. K., Boneau, C. A., Burstein, K. R., \& Pennypacker, H. S. Positive and negative generalization gradients obtained after equivalent training conditions. Journal of Comparative and Physiological Psychology, 1963, 56, 111-116.

Richards, R. W., \& Marcattilio, A. J. Stimulus control and delayed reinforcement. Learning \& Motivation, 1978, 9, 54-68.

Rilling, M. Stimulus control and inhibitory processes. In W. K. Honig \& J. E. R. Staddon (Eds.), Handbook of operant behavior. Englewood Cliffs, N.J: Prentice-Hall, 1977.

Sutherland, N. S., \& Mackintosh, N. J. Mechanisms of animal discrimination learning. New York: Academic Press, 1971.

Terrace, H. S. By-products of discrimination learning. In G. H. Bower \& J. T. Spence (Eds.), Learning and motivation (Vol. 5). New York: Academic Press, 1972.

Thomas, D. R., Svinicki, M. D., \& Svinicki, J. G. Masking of stimulus control during generalization testing. Journal of Experimental Psychology, 1970, 84, 479-482.

(Received for publication October 24, 1978; accepted December 12, 1978.) 